\title{
P-body components, Dhh1 and Pat1, are involved in tRNA nuclear-cytoplasmic dynamics
}

\author{
REBECCA L. HURTO and ANITA K. HOPPER \\ Department of Molecular Genetics, The Center for RNA Biology, The Ohio State University, Columbus, Ohio 43210, USA
}

\begin{abstract}
The nuclear-cytoplasmic distribution of tRNA depends on the balance between tRNA nuclear export/re-export and retrograde tRNA nuclear import in Saccharomyces cerevisiae. The distribution of tRNA is sensitive to nutrient availability as cells deprived of various nutrients exhibit tRNA nuclear accumulation. Starvation induces numerous events that result in translational repression and P-body formation. This study investigated the possible coordination of these responses with tRNA nuclearcytoplasmic distribution. Dhh1 and Pat1 function in parallel to promote translation repression and P-body formation in response to starvation. Loss of both, Dhh1 and Pat1, results in a failure to repress translation and to induce P-body formation in response to glucose starvation. This study reports that nutrient deprived $d h h 1$ pat1 cells also fail to accumulate tRNA within nuclei. Conversely, inhibition of translation initiation and induction of P-body formation by overproduction of Dhh1 or Pat1 cause tRNA nuclear accumulation in nutrient-replete conditions. Also, loss of the mRNA decapping activator, Lsm1, causes tRNA nuclear accumulation. However, the coordination between P-body formation, translation repression, and tRNA distribution is limited to the early part of the P-body formation/translation repression pathway as loss of mRNA decapping or $5^{\prime}$ to 3' degradation does not influence tRNA nuclear-cytoplasmic dynamics. The data provide the first link between P-body formation/translation initiation and tRNA nuclear-cytoplasmic dynamics. The current model is that Dhh1 and Pat1 function in parallel to promote starvation-induced tRNA nuclear accumulation.
\end{abstract}

Keywords: Prt1; Lsm1; starvation; translation; nutrient availability; nuclear import and export

\section{INTRODUCTION}

tRNA, which are transcribed in the nucleus, must be exported to the cytoplasm in order to perform the essential function of transferring amino acids to the growing peptide chain during protein synthesis. Surprisingly, studies have demonstrated that tRNA transit from the cytoplasm into the nucleus (Shaheen and Hopper 2005; Takano et al. 2005). The imported tRNA can be re-exported to the cytoplasm (Whitney et al. 2007). However, the normal cellular function(s) for tRNA nuclear import/re-export is not yet known. Shuttling mature tRNA in and out of the nucleus appears to be a conserved process as it has been shown to occur in yeast (Shaheen and Hopper 2005; Takano et al. 2005; Whitney et al. 2007), rat hepatoma (Shaheen et al. 2007), and HeLa cells (Zaitseva et al. 2006). Additionally, it has been proposed that

Reprint requests to: Anita K. Hopper, Department of Molecular Genetics, The Center for RNA Biology, The Ohio State University, 484 W. 12th Ave., Columbus, OH 43210, USA; e-mail: hopper.64@osu.edu; fax: (614) 292-4466.

Article published online ahead of print. Article and publication date are at http://www.rnajournal.org/cgi/doi/10.1261/rna.2558511.
HIV has usurped tRNA nuclear import pathway(s) as one means of allowing entry of retrotranscribed complexes into nuclei of nondividing vertebrate cells (Zaitseva et al. 2006).

tRNA nuclear-cytoplasmic transit appears to be dynamically regulated as cells exhibit tRNA nuclear accumulation when acutely deprived of amino acids (Sarkar et al. 1999; Grosshans et al. 2000; Shaheen and Hopper 2005), glucose (Whitney et al. 2007), or phosphate (Hurto et al. 2007), in contrast to cells grown in nutrient-rich conditions that exhibit an even distribution of tRNA throughout the nucleus and cytoplasm. Recent data have shown that tRNA transit into the nucleus is a constitutive process (Murthi et al. 2010). Therefore, the rates of primary export, re-export, and import of tRNA are likely balanced by regulatory pathways that result in the even distribution or nuclear accumulation of tRNA. While conditions that induce tRNA nuclear accumulation (e.g., starvation) or a return to an even cellular distribution (e.g., re-feeding) have been identified, the mechanisms for the regulation of tRNA nuclearcytoplasmic distribution remain unclear.

The current model of tRNA nuclear-cytoplasmic dynamics involves three movements: primary nuclear export of 
newly transcribed pre-tRNA, retrograde transport (nuclear import) of mature tRNA from the cytoplasm to the nucleus, and re-export of previously imported tRNA back to the cytoplasm (for review, see Phizicky and Hopper 2010). The budding yeast, Saccharomyces cerevisiae, is well suited to study these processes because primary export can be easily distinguished from re-export in these cells. The characteristic of $S$. cerevisiae that allows one to distinguish primary export and re-export is the mitochondrial location of the tRNA splicing endonuclease complex (Yoshihisa et al. 2003). This complex, which removes the intron from newly transcribed end-processed pre-tRNA, is located on the cytoplasmic surface of mitochondria (Yoshihisa et al. 2003, 2007). Thus, if there is a defect in primary tRNA export, end-processed intron-containing pre-tRNA will accumulate because the nuclear tRNA are unable to access the mitochondrially located splicing complex. In contrast, a defect in re-export does not cause accumulation of intron-containing pre-tRNA, because the imported tRNA have already been spliced.

Los1 exports tRNA from the nucleus to the cytoplasm in S. cerevisiae (Hopper et al. 1980; Hellmuth et al. 1998; Sarkar and Hopper 1998). The role of Los1 in tRNA export is conserved in many, but not all, organisms. Xpo-t, the Los1 vertebrate homolog, efficiently binds end-processed, intron-containing pre-tRNA and mature tRNA (Arts et al. 1998b; Lipowsky et al. 1999; Cook et al. 2009). Orthologs of Los1 in plants (PAUSED) (Hunter et al. 2003) and vertebrates (Xpo-t) (Arts et al. 1998a; Kutay et al. 1998) participate in tRNA nuclear export. Deletion of LOS1 causes increased nuclear pools of tRNA, observed by fluorescence in situ hybridization (FISH), and accumulation of intron-containing pre-tRNA observed by Northern analysis. It is the only nuclear export protein known to participate in the primary tRNA export pathway that transports intron-containing pre-tRNA to the cytoplasm (Murthi et al. 2010). As Los1 is unessential, in yeast, at least one additional nuclear exporter of intron-containing pretRNA must exist. Recently, Los1 has been shown to participate in the re-export of mature tRNA, in S. cerevisiae, in addition to primary tRNA export (Murthi et al. 2010).

Re-export of previously imported mature tRNA to the cytoplasm is also mediated by Msn5. The mamallian (Exp5) and Drosophila (dmExp5) orthologs of Msn5 have also been implicated in tRNA transport (Bohnsack et al. 2002; Calado et al. 2002; Shibata et al. 2006). Defects in tRNA re-export can be monitored by genetic and biochemical means. Similar to primary export defects, cells containing a mutation causing the re-export defect should exhibit nuclear accumulation of spliced tRNA; however, this accumulation is dependent on tRNA nuclear import. Loss of MSN5 causes import-dependent tRNA nuclear accumulation without causing an increase in the steady state levels of intron-containing pre-tRNA. Therefore, Msn5 participates in the tRNA re-export pathway, but not primary tRNA export (Eswara et al. 2009; Murthi et al. 2010). As msn5s los $1 \Delta$ double mutant cells are viable, one or more additional tRNA re-export pathways may exist (Takano et al. 2005; Murthi et al. 2010).

Mtr10, the S.cerevisiae homolog of the vertebrate TRN$\mathrm{SR} 2$, is required for nuclear accumulation of previously cytoplasmic, mature tRNA as mtr10A cells fail to exhibit starvation-induced tRNA nuclear accumulation (Shaheen and Hopper 2005). Although this lack of accumulation could be due to a defect in nuclear retention of tRNA, the genetic data argue that a failure to import is the more likely mechanism (Murthi et al. 2010). If Mtr10 participates in tRNA nuclear import, then it would be expected that loss

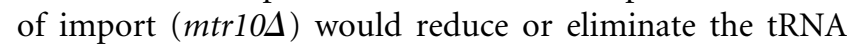
nuclear accumulation resulting from a re-export defect. As $m \operatorname{tr} 10 \Delta m s n 5 \Delta$ and $m \operatorname{tr} 10 \Delta$ los $1 \Delta$ cells fail to exhibit tRNA nuclear accumulation, regardless of nutritional status, Mtr10 is likely to directly or indirectly mediate tRNA nuclear import (Murthi et al. 2010). Consistent with this model, mtr10s los1s cells have a tRNA splicing defect similar to los1s cells (Murthi et al. 2010). As Mtr10 has been shown to import proteins such as $\mathrm{Npl} 3$ into the nucleus (Pemberton et al. 1997) and lacks obvious RNA binding motifs, Mtr10 may import tRNA into the nucleus via an adapter protein. Retrograde transport of tRNA provides a mechanism by which spliced tRNA accumulates in nuclei.

Previous and current studies are directed at determining the mechanism(s) that regulates the distribution of tRNA between the nucleus and cytoplasm. Nuclear accumulation of tRNA in response to acute amino acid and glucose starvation is rapid ( $\sim 15 \mathrm{~min}$ ) and reversible (Whitney et al. 2007). Starvation-induced tRNA nuclear accumulation is not due to inhibition of primary tRNA export as introncontaining pre-tRNA fail to accumulate and transcription is not required (Whitney et al. 2007). Moreover, it is dependent on retrograde movement of tRNA as $m$ tr10 10 cells exhibit an even distribution of tRNA regardless of nutritional status. Therefore, the starvation-induced tRNA nuclear accumulation is the result of decreased re-export, increased import, or a combination of both.

As tRNA nuclear accumulation occurs in response to starvation, other nutrient responsive pathways were evaluated to determine if they influence tRNA nuclear-cytoplasmic distribution. Previous studies revealed that tRNA nuclear accumulation is independent of the general amino acid control pathway as gcn $2 \Delta$ (eIF2 kinase) cells are not blocked for starvation-induced tRNA nuclear accumulation (Whitney et al. 2007). TOR has an undefined role in tRNA nuclear-cytoplasmic dynamics. Treatment of cells with rapamycin causes phenotypes associated with starvation (Barbet et al. 1996). However, rapamycin treatment failed to cause the tRNA nuclear accumulation that was expected under this pseudostarvation condition (Whitney et al. 2007). Two additional pathways that have been examined are PKA, using the low, but constitutively active mutant, $t p k^{w 1} t p k 2 \Delta$ 


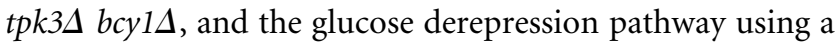
reg1s strain, which is constitutively derepressed. These strains are not only defective for PKA signaling and glucose repression, respectively, but exist in a constitutively stressed state. These strains are blocked for starvation-induced tRNA nuclear accumulation. However, the lack of tRNA nuclear accumulation in these strains may be due to their inability to experience acute starvation signaling (Whitney et al. 2007). The previous cumulative data do not provide a model for starvation-induced tRNA nuclear accumulation.

In order to gain additional insight into the regulation of tRNA nuclear-cytoplasmic distribution, other cellular processes that have rapid and reversible responses to acute nutrient deprivation were examined. Similar to tRNA subcellular dynamics, P-body formation and translational repression are conserved processes that occur within 10-15 min in response to starvation and can be reversed by the addition of the missing nutrient (Brengues et al. 2005). The number and size of P-bodies (mRNP aggregates) increase rapidly in response to acute glucose starvation. P-bodies contain many proteins involved in mRNA decay including Dhh1 (Rck/p54/DDX6), Pat1 (PatL1/Pat1b/HPat), Edc1-3, Lsm1-7, Dcp1, Dcp2, and Xrn1/Kem1 (Buchan and Parker 2009). Interestingly, Dhh1 and Xrn1 are shared with other mRNP aggregates, such as stress granules (Buchan and Parker 2009). Although P-body formation is very complicated and in the process of being fully described, loss-offunction mutants and overexpression of some proteins have been identified that block starvation-induced P-body formation or cause increased P-body formation independent of nutritional status. Many of these mutants and overexpressed gene products also influence translation.

As nutrient deprivation induces numerous signaling events, including translational repression and P-body formation, it is possible that tRNA nuclear accumulation may be coordinated with these processes. The nutrientresponsive translational repressors and decapping activators, Dhh1 and Pat1, function in parallel to promote starvationinduced increases in P-body formation and decreases in translation (Coller and Parker 2005). This study reports that either Dhh1 or Pat1 is required for starvation-induced tRNA nuclear accumulation as the double mutant, dhh1s pat1s, fails to exhibit tRNA nuclear accumulation when deprived of amino acids or glucose. Conversely, overexpression of either Dhh1 or Pat1 causes tRNA nuclear accumulation in cells that are provided with all necessary nutrients. Loss of another P-body component, Lsm1 $(l s m 1 \Delta)$, results in increased P-body formation (Sheth and Parker 2003), as well as tRNA nuclear accumulation. In contrast, loss of decapping activity or $5^{\prime}$ to $3^{\prime}$ exonuclease activity $(x r n 1 \Delta)$ did not influence tRNA nuclearcytoplasmic dynamics. Cumulatively, the data indicate that Dhh1 and Pat1 function in parallel to promote nutrient responsive P-body formation and tRNA nuclear accumulation. The data also indicate that there may be an additional undescribed pathway, which is Pat1/Dhh1 independent, which contributes to the tRNA nuclear accumulation observed when translation initiation is disrupted. This work provides the first evidence that P-body formation and translation initiation are coordinated with tRNA nuclear-cytoplasmic distribution.

\section{RESULTS}

\section{Dhh1 and Pat1 regulate tRNA nuclear-cytoplasmic distribution}

P-body formation/translation repression and tRNA nuclear-cytoplasmic redistribution are responses to nutrient deprivation. Two main approaches were utilized to evaluate whether starvation-induced P-body formation and translation repression are coordinated with starvation-induced tRNA nuclear accumulation. The first approach was to block increases in P-body formation and decreases in translation observed during nutrient deprivation and then determine the distribution of tRNA between the nucleus and the cytoplasm. The second approach was to induce P-body formation and/or translation repression and then determine the cellular distribution of tRNA. In pursuing the first approach, tRNA subcellular dynamics were evaluated in $d h h 1 \Delta$, pat $1 \Delta$, and dhh1s pat1s double mutant yeast strains.

Dhh1 is an mRNA decapping activator and starvationinduced translation repressor that inhibits $48 \mathrm{~S}$ formation (Coller and Parker 2005). Pat1 is a starvation-induced translation repressor that limits $48 \mathrm{~S}$ production and a decapping activator that directly stimulates the mRNA decapping activity of the Dcp1/Dcp2 complex (Nissan et al. 2010). Dcp1/Dcp2 is the complex that removes $5^{\prime}$ methyl caps from deadenylated mRNAs. Dhh1 and Pat1 function in parallel to repress translation in response to glucose or amino acid starvation (Holmes et al. 2004; Coller and Parker 2005), and to induce P-body formation in response to glucose starvation (Coller and Parker 2005). The distributions of P-body components are generally not studied under conditions of amino acid deprivation. Strains lacking either DHH1 or PAT1 have defects in mRNA decapping and small reductions in starvation-induced P-body formation and translation repression. The dhh1 pat1 double mutant neither forms P-bodies during glucose deprivation nor represses translation when deprived of amino acids or glucose (Coller and Parker 2005).

The subcellular distribution of tRNA in strains lacking DHH1 and/or PAT1 was determined using FISH. As acute deprivation of either amino acids or glucose induces tRNA nuclear accumulation in wild-type cells, all of the strains were evaluated under fed, amino acid deprived, and glucose deprived conditions (Fig. 1A). As expected, wild-type cells grown in nutrient-rich conditions exhibited an even distribution of tRNA throughout the cells (Fig. 1A, row 1). 


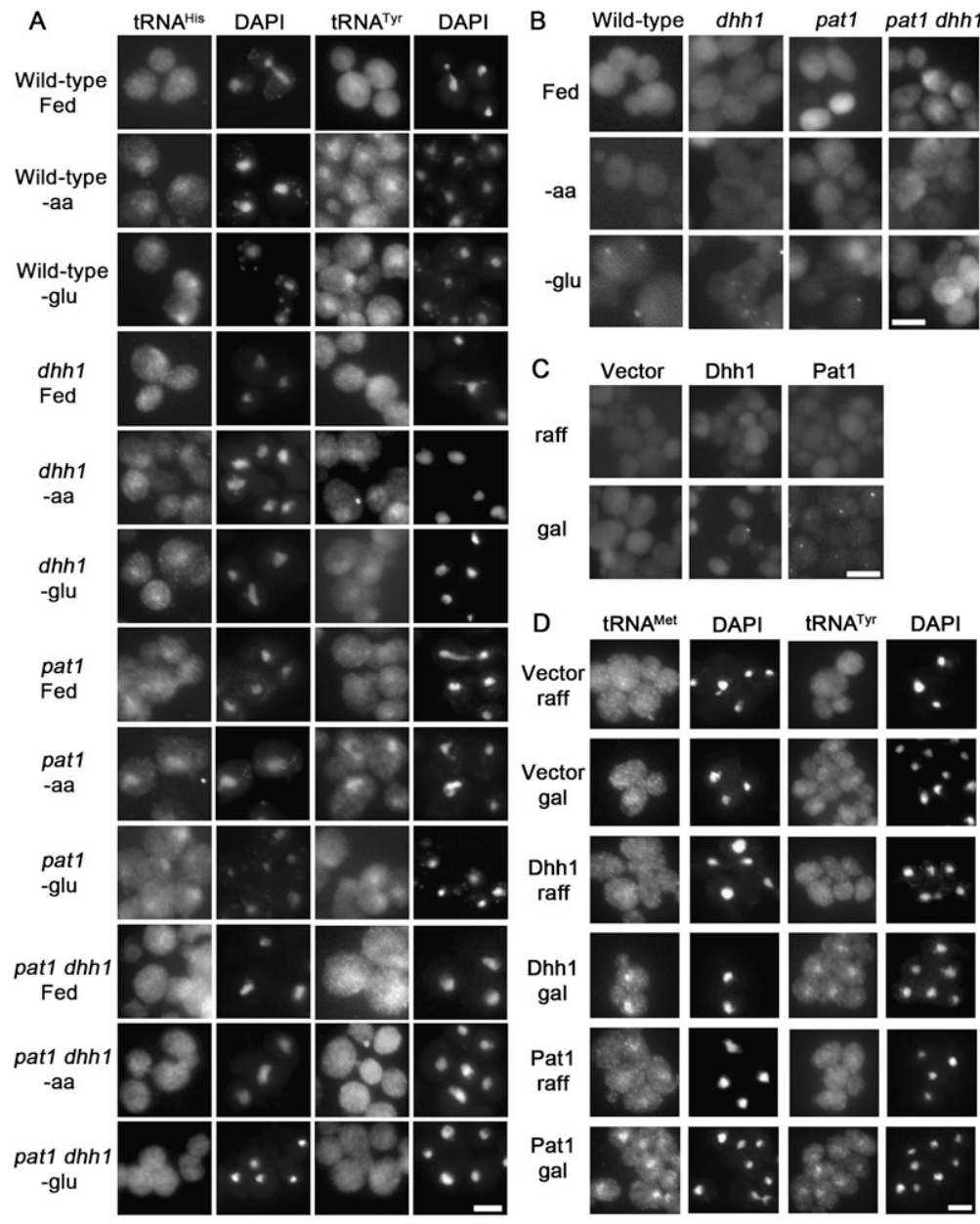

FIGURE 1. Evaluation of the roles of Dhh1 and Patl in tRNA nuclear-cytoplasmic distribution. (A) FISH analysis of wild-type, pat1, dhhl, and pat1 dhh1 cells during starvation. Cells were probed for the location of $\mathrm{tRNA}^{\text {His }}$ (column 1) or $\mathrm{tRNA}^{\mathrm{Tyr}}$ (column 3) after incubation in SC media (Fed), or in media lacking either amino acids (-aa) or glucose (-glu) for $2 \mathrm{~h}$. DNA was stained with DAPI to reveal the locations of nuclei (columns 2,4). (B) P-body formation upon nutrient deprivation: Wild-type, pat 1 , dhhl, and pat $1 \mathrm{dhh} 1$ cells with plasmidborne Dcp2-RFP after incubation in SC-ura media (Fed), or in media lacking either amino acids (-aa) or glucose (-glu) for $2 \mathrm{~h}$. (C) Distribution of plasmid-borne Dcp2-RFP in wild-type cells with vector alone, $\mathrm{P}_{\mathrm{GAL}}-\mathrm{DHH} 1$ (Dhh1), or $\mathrm{P}_{\mathrm{GAL}}-\mathrm{PAT1}$ (Pat1) grown in non-inducing media (raff; row 1) or after $3 \mathrm{~h}$ of induction by addition of $1 / 10$ volume of $20 \%$ galactose (gal; row 2). (D) Wild-type cells with empty vector, $\mathrm{P}_{\mathrm{GAL}}-D H H 1$ (Dhh1), or $\mathrm{P}_{\mathrm{GAL}}-P A T 1$ (Pat1) grown in media with raffinose (raff), or $3 \mathrm{~h}$ after the addition of $1 / 10$ volume of $20 \%$ galactose were probed for the location of tRNA ${ }^{\text {Met }}$ (column 1) or tRNA ${ }^{\text {Tyr }}$ (column 3) using FISH, and for the location of DNA (DAPI; columns 2,4). Bars are $5 \mu \mathrm{m}$.

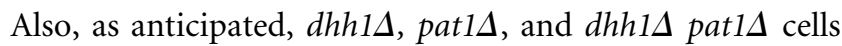
grown in nutrient-rich conditions exhibited an even distribution of tRNA throughout the cells (Fig. 1A, rows 4,7,10). As previously shown, wild-type cells undergoing amino acid (Sarkar et al. 1999; Grosshans et al. 2000) or glucose starvation (Whitney et al. 2007) exhibited tRNA nuclear accumulation as indicated by the increased tRNA (FITC) signal that co-localizes with the DAPI stained DNA signal (Fig. 1A, rows 2,3, respectively). In contrast, tRNA did not accumulate in the nuclei of $\operatorname{dhh} 1 \Delta$ pat $1 \Delta$ cells deprived of amino acids (Fig. 1A, row 11) or glucose (Fig. 1A, row 12).
The single mutants, dhh1s and pat $1 \Delta$, subjected to deprivation of amino acids (Fig. 1A, rows 5,8, respectively) or glucose (Fig. 1A, rows 6,9, respectively) exhibited less tRNA nuclear accumulation compared to wild-type cells. This was anticipated because dhhls and pat1s single mutations produce milder defects in P-body formation and translation repression during acute glucose withdrawal than the double mutant does (Coller and Parker 2005). Therefore, mutations that inhibit starvationinduced P-body formation and translation repression also inhibit starvationinduced tRNA nuclear accumulation.

As the standard conditions utilized for FISH studies of tRNA subcellular distribution (defined media, $0.15<A_{600}$ $<0.35,23^{\circ} \mathrm{C}$, and 2 -h starvation) differ from the standard conditions reported to evaluate P-body formation (complex media, $A_{600}>0.4,30^{\circ} \mathrm{C}$, and 10-min starvation), experiments were undertaken to determine if P-bodies form in conditions identical to those employed for FISH. This is important as P-body formation and tRNA nuclearcytoplasmic distribution are sensitive to cell density and culture conditions (Whitney et al. 2007; Nissan and Parker 2008). When cell density increases, tRNA distribution becomes increasingly nuclear (Whitney et al. 2007) and P-bodies become larger and more numerous (Brengues et al. 2005; Nissan and Parker 2008).

If differences in media, density, temperature, and length of starvation between the two types of analyses do not significantly change the overall pattern of starvation-induced P-body formation, then plasmid-borne Dcp2-RFP (and other P-body markers) should exhibit increased aggregation in glucose-starved wild-type, $d h h 1 \Delta$, and pat $1 \Delta$ cells, but not exhibit increased aggregation in dhh1s pat1s cells when assessed using the conditions employed for FISH. Dcp2 was selected for use as a P-body marker for this work because current data indicate that it is still exclusive to P-bodies in S. cerevisiae (Buchan and Parker 2009; Buchan et al. 2010). Very few of

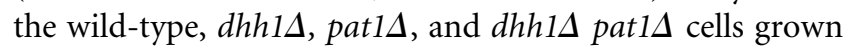
in media containing amino acids and glucose showed 1 or more Dcp2-RFP foci (Fig. 1B, row 1; Table 1). No change in Dcp2-RFP aggregation was observed when wild-type, 
TABLE 1. Percent of cells containing Dcp2-RFP foci

\begin{tabular}{llcl}
\hline Strain & Media & \% cells $^{\mathrm{a}}$ & SD $^{\mathrm{b}}$ \\
\hline Wild type & SC & 1.1 & 0.9 \\
Wild type & -aa & 1.7 & 1.5 \\
Wild type & -glu & 75 & 7.7 \\
dhh1 & SC & 0.4 & 0.3 \\
dhh1 & -aa & 2.3 & 1.1 \\
dhh1 & -glu & 58.6 & 2.3 \\
pat1 & SC & 0.2 & 0.3 \\
pat1 & -aa & 0.2 & 0.4 \\
pat1 & -glu & 47 & 20 \\
dhh1 pat1 & SC & 1.8 & 1.8 \\
dhh1 pat1 & -aa & 1.5 & 1.1 \\
dhh1 pat1 & -glu & 1.2 & 0.8 \\
\hline
\end{tabular}

a Percent of cells with one or more visible Dcp2-RFP foci as determined by counting 100 or more cells from three or more independent cultures. Cells were nutrient deprived, -aa or -glu, for $2 \mathrm{~h}$.

${ }^{\mathrm{b}}$ Standard deviation.

dhh1s, pat $1 \Delta$, and dhh1s pat1s cells were deprived of amino acids (Fig. 1B, row 2; Table 1). In contrast, a large increase in P-body formation was observed in wild-type cells deprived of glucose for $2 \mathrm{~h}(1.1 \%$ vs. $75 \%$ of cells with 1 or more Dcp2-RFP foci) (Fig. 1B, column 1, cf. rows 1 and 3). Increases in Dcp2-RFP aggregation were also observed in $d h h 1 \Delta$ (0.4\% vs. $59 \%)$ (Fig. 1B column 2) and pat $1 \Delta$ ( $0.2 \%$ vs. $47 \%)$ (Fig. 1B column 3 ) cells starved for glucose for $2 \mathrm{~h}$ The double mutant, pat $1 \Delta$ dhh $1 \Delta$, did not exhibit an increase in Dcp2-RFP aggregation $(1.8 \%$ vs $1.2 \%$ ) (Fig. 1B column 4) when glucose was withdrawn for $2 \mathrm{~h}$ Thus, despite the differences in cell density, media, and duration of starvation, wild-type, dhh1s, pat $1 \Delta$, and dhh1s pat $1 \Delta$ cells demonstrated the expected pattern of Dcp2-RFP foci occurrence.

Overexpression of Dhh1 or Patl causes increased formation of Dcp2-containing P-bodies (Fig. 1C; Coller and Parker 2005) and translation repression in wild-type cells provided all necessary nutrients (Coller and Parker 2005). To determine whether overexpression of Dhh1 or Pat 1 affects tRNA nuclear-cytoplasmic distribution, wild-type cells containing plasmids expressing either Dhh1 or Pat1 under the control of a Gal promoter were evaluated. Wildtype cells grown under noninducing conditions did not exhibit tRNA nuclear accumulation (Fig. 1D, row 1). After $3 \mathrm{~h}$ of galactose induction, cells overexpressing Dhh1 (Fig. 1D, row 4) or Pat1 (Fig. 1D, row 6) exhibited tRNA nuclear accumulation. This nuclear accumulation was not due to the addition of galactose to the raffinose-containing media as cells with vector alone did not exhibit tRNA nuclear accumulation (Fig. 1D, row 2). As overexpression of Dhh1 or Pat1 did not cause accumulation of intron-containing tRNA (Fig. 2), a hallmark of defects in primary tRNA export (Shaheen and Hopper 2005; Takano et al. 2005; Whitney et al. 2007), the tRNA nuclear accumulation observed is due to retrograde import and/or blocked reexport of mature tRNA. The result supports the model that tRNA nuclear-cytoplasmic distribution is coordinately regulated with P-body formation and translation repression.

Pat1 has separable domains that mediate its many activities: interactions with Lsm1, Dcp2, and Edc3, P-body formation, and translation repression (Pilkington and Parker 2008). Pat1 is also able to mediate starvation-induced tRNA nuclear accumulation in the absence of Dhh1 (Fig. 1A, rows 5,6). Amino acids $254-422$ of Pat1 are required for its decapping activation activity, whereas amino acids 422-697 are required for translation repression and increased P-body formation during overexpression, and for recruitment of Lsm1 and Dcp2 to P-bodies (Pilkington and Parker 2008). In order to determine which domain of Pat1

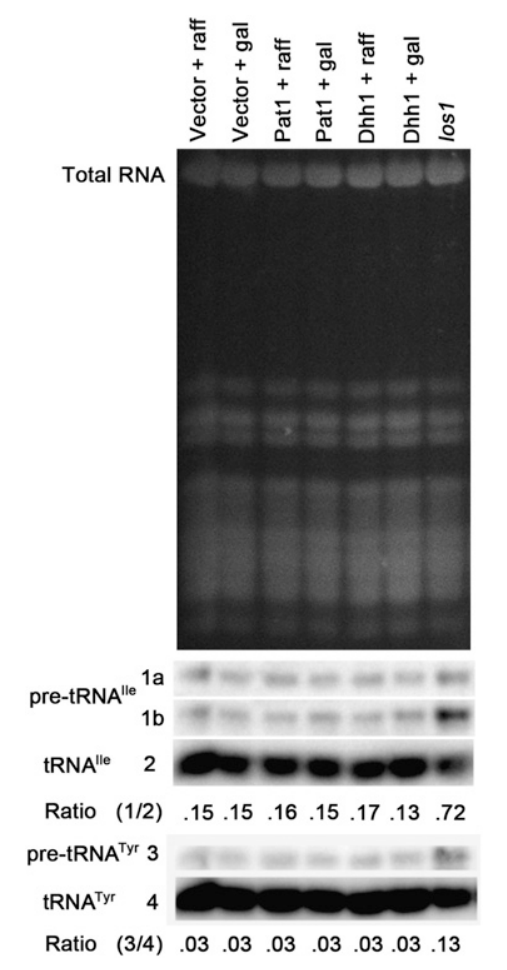

FIGURE 2. tRNA nuclear accumulation caused by overexpression of Dhh1 or Pat1 is due to tRNA nuclear import and/or defective reexport, but not due to a block in initial tRNA nuclear export. Northern analysis of RNA extracted from los 1 cells or wild-type cells with vector alone, $\mathrm{P}_{\mathrm{GAL}}-D H H 1$ (Dhh1), or $\mathrm{P}_{\mathrm{GAL}}-P A T 1$ (Pat1) grown in media with raffinose (raff), or $3 \mathrm{~h}$ after the addition of $1 / 10$ volume of $20 \%$ galactose. Wild-type with pRS416 serves as a negative, normal distribution control, whereas los 1 serves as positive, intron-containing pre-tRNA accumulation control. The top panel is ethidium bromide stained gel prior to transfer that reveals the RNAs present and documents equivalent loading of RNA in each lane. The next three panels are a Northern blot probed using a ${ }^{32} \mathrm{P}$ labeled oligo that detects tRNA $^{\text {Ile }}$ ( $1 a$-initial transcript of pre-tRNA ${ }^{\text {Ile }}$; $1 b$-endprocessed, intron-containing pre-tRNA ${ }^{\text {Ile }}, 2$-mature tRNA $\left.{ }^{\text {Ile }}\right)$. The ratios of total precursor tRNA ${ }^{\mathrm{Ile}}(1 a+1 b)$ to mature tRNA ${ }^{\text {Ile }}$ is presented. A probe detecting tRNA ${ }^{\text {Tyr }}$ (3-intron-containing pre-tRNA ${ }^{\text {Tyr }}$, 4 - mature tRNA ${ }^{\mathrm{Tyr}}$ ) was employed in the bottom two panels. The ratios of total precursor tRNA ${ }^{\mathrm{Tyr}}$ to mature tRNA ${ }^{\mathrm{Tyr}}$ is presented. 
is important for tRNA nuclear accumulation, dhh1s pat1s cells containing low-copy plasmids encoding either pat $1 \Delta 254-422$ or pat $1 \Delta 422-697$ with native promoters were subjected to glucose starvation and then analyzed by FISH. Wild-type and dhh1s pat1s cells containing any of the Pat1 constructs exhibited the expected even distributions of tRNA when grown in nutrient-rich media (Fig. 3A, row 1). As expected, tRNA accumulate in the nuclei of wild-type (Fig. 3A, row 3, column 1) and dhh1s pat1s cells with full-length PAT1 (Fig. 3A, row 3, column 2) when undergoing glucose starvation. Similarly, dhh1s pat1s cells with pat1 $\Delta 254-422$ undergoing glucose starvation exhibited tRNA nuclear accumulation (Fig. 3A, row 3, column 3),

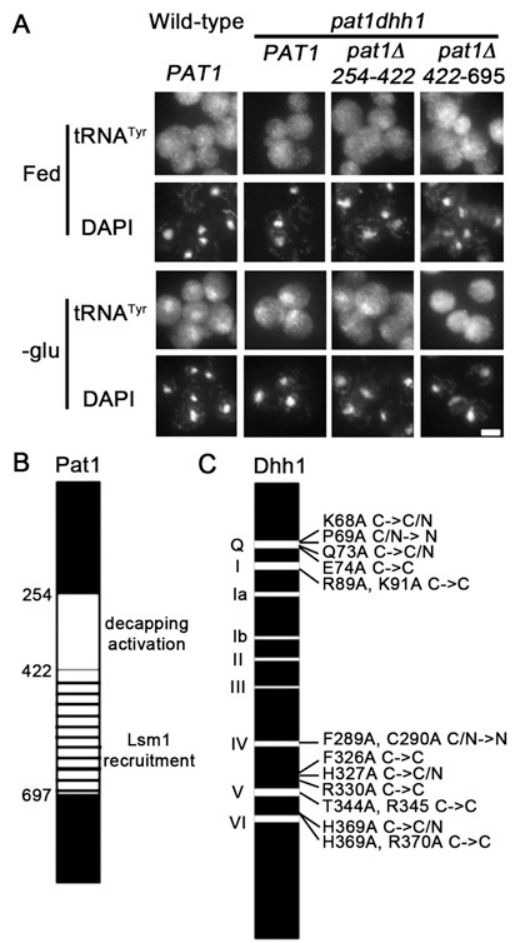

FIGURE 3. The decapping domain of Pat1 is unessential for its role in starvation-induced tRNA nuclear accumulation. $(A)$ Intracellular distribution of $\mathrm{tRNA}^{\mathrm{Tyr}}$ (rows 1,3) and DNA (DAPI; rows 2,4) in wild-type cells and in pat $1 \mathrm{dhh} 1$ cells with plasmid-borne full-length

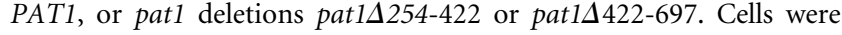
incubated in SC-ura (Fed) or in media lacking glucose (-glu) for $2 \mathrm{~h}$ Bar depicts $5 \mu \mathrm{m}$. (B) Diagram of Pat1 including deleted domains from amino acids 254-422, and 422-697. (C) Pictorial representation of mutations that influenced the ability of Dhh1 to complement dhh1s pat $1 \Delta$ cells loss of starvation-induced tRNA nuclear accumulation. Each mutation is listed by original amino acid (single letter), number in amino acid sequence, replacement amino acid (single letter), followed by phenotypic indicator. $\mathrm{C} \rightarrow \mathrm{C}$ indicates cells maintained an even distribution of tRNA in fed and in glucose starvation conditions (no complementation). $\mathrm{C} \rightarrow \mathrm{C} / \mathrm{N}$ indicates cells maintained an even distribution of tRNA in fed conditions, but exhibited a lower than expected tRNA nuclear accumulation phenotype in glucose starvation conditions (poor complementation). $\mathrm{C} / \mathrm{N} \rightarrow \mathrm{N}$ indicates that low levels of tRNA nuclear accumulation were observed in fed conditions. indicating that the decapping activation activity of Pat 1 is not required for tRNA nuclear accumulation in response to nutrient deprivation (Fig. 3B). In contrast, dhh1s pat1s cells with pat1 $\Delta 422-697$ undergoing glucose starvation exhibited an even distribution of tRNA (Fig. 3A, row 3, column 4). The failure of pat1 $\Delta 422-697$ to restore starvation-induced tRNA nuclear accumulation in dhh1s pat1s cells indicates that this domain is important for tRNA subcellular dynamics (Fig. 3B). As the domain of Pat1 that is important for translation repression and increased P-body formation is also important for starvation-induced tRNA nuclear accumulation, the data support the model of coordinate regulation of these processes.

Dhh1 is an RNA binding protein containing nine DEAD box conserved sequence motifs that shares sequence homology with eIF4A (Cheng et al. 2005). Dhh1 has several activities. The steady-state level of EDC1 mRNA is an indicator of Dhh1 decapping activator activity as deletion of $\mathrm{DHH} 1$ causes an increase of its steady-state level (Cheng et al. 2005). Dhh1 is able to mediate starvation-induced tRNA nuclear accumulation in the absence of Pat1 because pat $1 \Delta$ cells exhibit starvation-induced tRNA nuclear accumulation when dhh1s pat1s cells do not (Fig. 1A, rows $8,9)$.

In order to determine which domain(s) of Dhh1 is important for tRNA nuclear accumulation, dhh1s pat1s cells were transformed with low-copy plasmids containing Dhh1 constructs with the glyceraldehyde-3-phosphate dehydrogenase promoter and various mutations (Cheng et al. 2005); the resulting cells were either kept in rich media or were subjected to glucose starvation and then analyzed by FISH. Four different types of results were obtained (Fig. 3C; Supplemental Table 1). Six different plasmid-borne, dhh1 mutants failed to restore tRNA nuclear accumulation to glucose deprived dhh1s pat $1 \Delta$ cells (Fig. 3C, C $\rightarrow$ C). Four additional plasmid-borne, dhh1 mutants showed reduced ability to complement the defective starvation-induced tRNA nuclear accumulation in dhh1s pat1s cells (Fig. $3 \mathrm{C}, \mathrm{C} \rightarrow \mathrm{C} / \mathrm{N})$. In contrast, four plasmid-borne $d h h 1$ mutants caused increased nuclear levels of tRNA in dhh1s pat1s cells grown in nutrient-rich conditions (Fig. 3C, $\mathrm{C} / \mathrm{N} \rightarrow \mathrm{N})$. Ten plasmid-borne $d h h 1$ mutants complemented the inability of dhh1s pat1s cells to exhibit starvationinduced tRNA nuclear accumulation (Supplemental Table 1). Some of the $d h h 1$ mutants used in this study have been evaluated for RNA binding activity and impact on steadystate levels of EDC1 mRNA (Cheng et al. 2005). The ability of a plasmid-borne dhh1 mutant to restore starvationinduced tRNA nuclear accumulation in dhh1s pat1s cells did not correlate with defects in RNA binding activity or alteration of EDC1 mRNA steady-state levels. Several mutations that are defective in starvation-induced tRNA nuclear accumulation occur at or near the salt bridge between motif $\mathrm{V}$ and motif $\mathrm{I}$ and in the $\mathrm{Q}$ motif that stacks against motif $\mathrm{V}$ and motif $\mathrm{I}$ (aa 74, 344, 330, 326) 
(Fig. 3C). The domain(s) or functions of Dhh1 that are important for starvation-induced tRNA accumulation remain unclear.

\section{Role of mRNA decay in tRNA nuclear-cytoplasmic dynamics}

Loss-of-function mutations of several mRNA decay pathway components cause increased P-body formation independent of nutrient deprivation. Lsm1 is found in complexes containing Lsm2-7, Dcp1, Dcp2, and Pat1, and it preferentially binds deadenylated mRNAs (Tharun and Parker 2001). Deletion of LSM1 causes increased P-body formation without causing defects in translation initiation as determined by polysome analysis (Sheth and Parker 2003; Holmes et al. 2004; Coller and Parker 2005). FISH was employed to determine the consequences of loss of Lsm1 function upon tRNA nuclear-cytoplasmic distribution. In contrast to the even distribution of tRNA exhibited by wild-type cells (Fig. 4A, column 1), $l s m 1 \Delta$ cells grown in nutrient-rich conditions exhibited tRNA nuclear accumulation (Fig. 4A, column 3). Therefore, Lsm1 has a role in maintaining an even distribution of tRNA within cells grown in nutrient-rich conditions.

To evaluate whether the tRNA nuclear accumulation observed in $l s m 1 \Delta$ cells depends on Dhh1 and Pat1, the triple mutant, $l s m 1 \Delta$ pat $1 \Delta$ dhh $1 \Delta$ was generated. If tRNA nuclear accumulation observed in $l \operatorname{sm} 1 \Delta$ cells depends upon Dhh1/Pat1, then $l s m 1 \Delta$ pat $1 \Delta$ dhhls cells should fail to exhibit tRNA nuclear accumulation. However, if this accumulation is independent of Dhh1/Pat1, then $l s m 1 \Delta$ pat1s dhh1s cells will exhibit tRNA nuclear accumulation. The results demonstrate that the tRNA nuclear accumulation observed in $l s m 1 \Delta$ cells is dependent on Dhh1 and

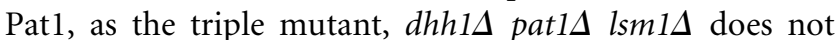
exhibit tRNA nuclear accumulation (Fig. 4A, column 4). Therefore, causing constitutive P-body formation, through deletion of the decapping activator, $L S M 1$, causes tRNA nuclear accumulation that is dependent on Dhh1/Pat1. The data further support co-regulation of P-body formation and tRNA nuclear-cytoplasmic distribution.

Pat1, Dhh1, and Lsm1 are involved in the early steps of P-body formation and mRNA decay, and, as shown here, tRNA subcellular dynamics. P-body components involved in the later steps of mRNA decay include the decapping complex, Dcp1 and Dcp2, and the $5^{\prime}$ to $3^{\prime}$ exonuclease, Xrn1. Mutations that cause loss of decapping, such as $d c p 2-7$, or loss of exonuclease activities, such as $x r n 1 \Delta$, also cause increased P-body formation (Fig. 4B,C; Sheth and Parker 2003; Holmes et al. 2004) and loss of starvation-induced translation repression (Holmes et al. 2004). To evaluate the influence of these later steps in P-body formation/mRNA decay upon tRNA nuclear-cytoplasmic distribution, $d c p 2-7$ cells and $x r n 1 \Delta$ cells cultured in nutrient-replete conditions were evaluated by FISH. If inducing formation of P-bodies

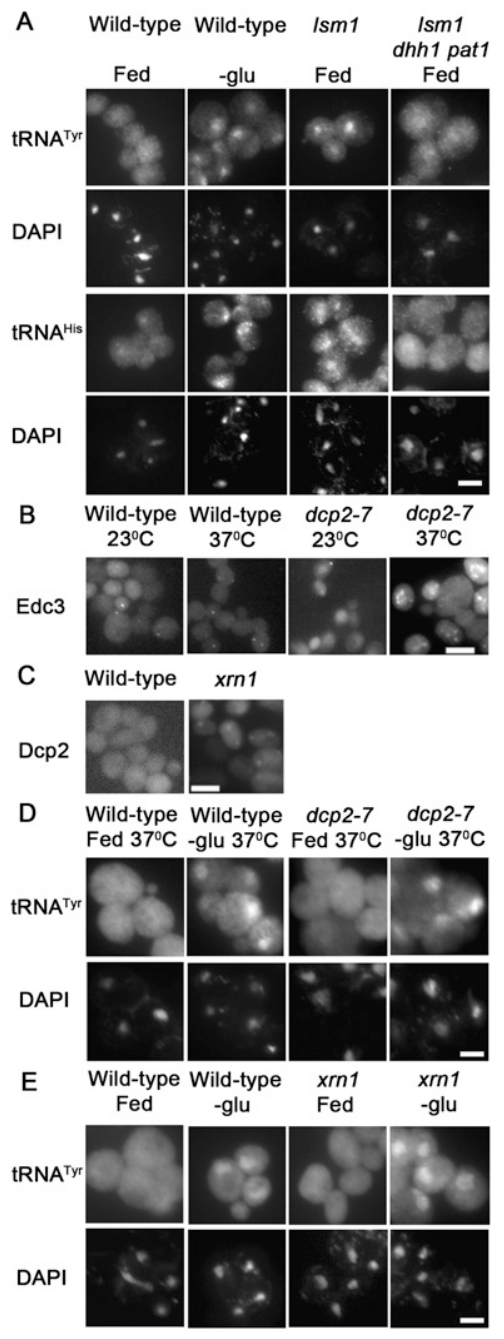

FIGURE 4. tRNA nuclear accumulation observed in $1 \mathrm{sm} 1$ cells is Dhh1/Pat1-dependent. (A) The distribution of tRNA ${ }^{\text {Tyr }}$ (row 1), tRNA $^{\text {His }}$ (row 3), or DNA (DAPI; rows 2,4 ) within wild-type, lsm1, and $l s m 1$ dhhl pat 1 cells grown on complete media and wild-type cells incubated $2 \mathrm{~h}$ in media lacking glucose were determined by FISH. (B) The distribution of plasmid-borne Edc3-mCherry within wild-type and $d c p 2-7$ cells at $23^{\circ} \mathrm{C}$ or after $2 \mathrm{~h}$ incubation at $37^{\circ} \mathrm{C}$. $(C)$ Wild-type and $x r n 1$ cells with plasmid-borne Dcp2-RFP. (D) Wild-type and $d c p 2-7$ cells incubated for $2 \mathrm{~h}$ at $37^{\circ} \mathrm{C}$ in media with (Fed) and without (-glu) glucose were analyzed by FISH. The probe to tRNA ${ }^{\text {Tyr }}$ (row 1) and DAPI staining (row 2) are presented. (E) The intracellular distributions of tRNA ${ }^{\text {Tyr }}$ (row 1) and DNA (DAPI; row 2) within wild-type and $x r n 1$ cells that were incubated for $2 \mathrm{~h}$ in media with (Fed) and without (-glu) glucose as revealed by FISH. Bars represent $5 \mu \mathrm{m}$.

by inhibiting the later steps in mRNA decay is sufficient to induce tRNA nuclear accumulation, then fed $d c p 2-7$ cells at nonpermissive temperature and fed $x r n 1 \Delta$ cells would exhibit tRNA nuclear accumulation. If tRNA nuclearcytoplasmic distribution is responsive to the early steps, but not the later steps in mRNA decay/P-body formation, then fed $d c p 2-7$ cells at nonpermissive temperature and xrn1s cells would not exhibit tRNA nuclear accumulation. At the permissive $\left(23^{\circ} \mathrm{C}\right)$ and the nonpermissive $\left(37^{\circ} \mathrm{C}\right)$ 
temperature, fed $d c p 2-7$ cells exhibited an even distribution of tRNA (Fig. 4D, column 3; data not shown) that was comparable to tRNA distribution in fed wild-type cells (Fig. 4D, column 1; Fig. 4E, column 1). Similarly, fed $x r n 1 \Delta$ cells exhibited an even distribution of tRNA (Fig. 4E, column 3). The data indicate that the later steps of mRNA decay, decapping, and exonucleolytic degradation do not influence tRNA nuclear-cytoplasmic distribution in nutrientreplete conditions. These results are in sharp contrast to the nuclear accumulation observed in $l s m 1 \Delta$ cells (Fig. 4A). Therefore, prior to the point of mRNA decapping, the increased formation of Dcp2 containing P-bodies is coordinated with tRNA nuclear accumulation, and at the point of mRNA decapping (Dcp2) and $5^{\prime}$ to $3^{\prime}$ decay (Xrn1), the correlation no longer exists.

A feature that $d c p 2$ and $x r n 1 \Delta$ cells have in common with dhh1s pat1s cells is loss of translation repression upon nutrient deprivation (Coller and Parker 2005). While $l s m 1 \Delta$ cells also share this feature, these cells already exhibit tRNA nuclear accumulation in fed conditions; therefore, evaluation of its tRNA nucleus-cytoplasmic distribution during starvation is not informative. If translation repression is required for nutrient responsive tRNA nuclear accumulation, then nutrient deprived $d c p 2-7$ cells, at the nonpermissive temperature would fail to exhibit tRNA nuclear accumulation. If translation repression is not required for starvation-induced tRNA nuclear accumulation, then glucose or amino acids deprivation would cause tRNA to accumulate within the nuclei of $d c p 2-7$ cells incubated at nonpermissive temperature. Wild-type and $d c p 2-7$ cells incubated $2 \mathrm{~h}$ at nonpermissive temperature in media lacking glucose or amino acids exhibited tRNA nuclear accumulation (Fig. 4D, columns 2,4 respectively; data not shown). Similarly, $x r n 1 \Delta$ cells incubated $2 \mathrm{~h}$ in media lacking glucose also exhibited tRNA nuclear accumulation (Fig. $4 \mathrm{E}$, row 4$)$. The data indicate that translation repression is not required for starvation-induced tRNA nuclear accumulation. In aggregate, the data indicate that tRNA nuclear-cytoplasmic distribution is coordinated with P-body formation until the point of mRNA decapping and decay, at which point the pathways become uncoupled.

\section{Loss of translation initiation causes tRNA nuclear accumulation}

Although translation repression is not required for starvation-induced tRNA nuclear accumulation, translation initiation may influence tRNA nuclear-cytoplasmic distribution. This possibility was considered because loss of translation initiation is not the same as translation repression. Translation repression occurs as part of a stress specific restricted translation program that varies depending on the type of stress (for review, see Spriggs et al. 2010), whereas temperature-sensitive mutations of translation initiation factors result in more general effects (Nielsen et al. 2004). The differences between the various stresses are further evidenced by the differences in P-body and stress granule formation (Buchan et al. 2010). Increased P-body formation is also observed when translation initiation is disrupted (Sheth and Parker 2003; Teixeira et al. 2005). This increase is specific to the initiation step of translation as disruption of elongation fails to induce increased P-body formation in yeast (Sheth and Parker 2003; Teixeira et al. 2005). Addition of the translation elongation inhibitor, cyclohexamide, also fails to induce tRNA nuclear accumulation in Xenopus oocytes (Lund and Dahlberg 1998). As inhibition of translation initiation causes P-body formation, it may also lead to tRNA nuclear accumulation.

The prt1-1 allele, a temperature-sensitive mutation of eukaryotic translation initiation factor $3 b$ (eIF3b), causes increased mRNA decay and decreased translation initiation at nonpermissive temperature (Barnes et al. 1993). Other temperature-sensitive alleles of $P R T 1$ have been shown to cause P-body formation at nonpermissive temperature (Brengues et al. 2005). Consistent with those studies, prt1-1 causes P-body formation at nonpermissive temperature as viewed by Dcp2-RFP (Fig. 5A, column 4), Edc3mCherry, and Pat1-GFP (data not shown). Wild-type cells grown at $23^{\circ} \mathrm{C}$ or for $2 \mathrm{~h}$ at $37^{\circ} \mathrm{C}$ exhibited an even distribution of tRNA (Fig. 5B, columns 1,2, respectively). The prt1-1 cells exhibited detectably higher levels of nuclear tRNA than wild-type cells at the permissive temperature $\left(23^{\circ} \mathrm{C}\right.$ ) (Fig. $5 \mathrm{~B}$, column 3 ) and prominent tRNA nuclear accumulation when incubated for $2 \mathrm{~h}$ at the nonpermissive temperature $\left(37^{\circ} \mathrm{C}\right)$ (Fig. $5 \mathrm{~B}$, column 4$)$. Northern analysis revealed that prt1-1 does not cause accumulation of introncontaining pre-tRNA (Fig. 6), a hallmark of primary tRNA export defects. Therefore, the tRNA nuclear accumulation observed in prt1-1 cells is due to nuclear import and/or blocked re-export of mature tRNA. To confirm this, tRNA nuclear import was blocked by mutation of MTR10 in prt1-1 cells and then the distribution of tRNA was assessed in prt1-1 mtr10s cells. At nonpermissive temperature, prt1-1 mtr104 cells fail to exhibit tRNA nuclear accumulation (Fig. 5C, column 4). Collectively, the data show that inhibition of translation initiation via prt1-1 causes nuclear accumulation of previously cytoplasmic, spliced tRNA.

To evaluate whether the tRNA nuclear accumulation observed in prt1-1 depends on Dhh1 and Pat1, the triple mutant, prt1-1 pat $1 \Delta$ dhh1s was generated. If the nuclear accumulation observed in prt1-1 cells depends upon Dhh1/ Pat1, then prt1-1 pat1s dhh1s cells would fail to exhibit tRNA nuclear accumulation at the nonpermissive temperature. However, if this accumulation is independent of Dhh1/Pat1, then prt1-1 pat1s dhh1s cells would exhibit tRNA nuclear accumulation similar to prt1-1 cells at the nonpermissive temperature. Surprisingly, when prt1-1 dhh1s pat $1 \Delta$ cells were incubated for $2 \mathrm{~h}$ at the nonpermissive temperature $\left(37^{\circ} \mathrm{C}\right)$, the cells exhibited detectable tRNA nuclear accumulation (Fig. 5C, column 3) that 


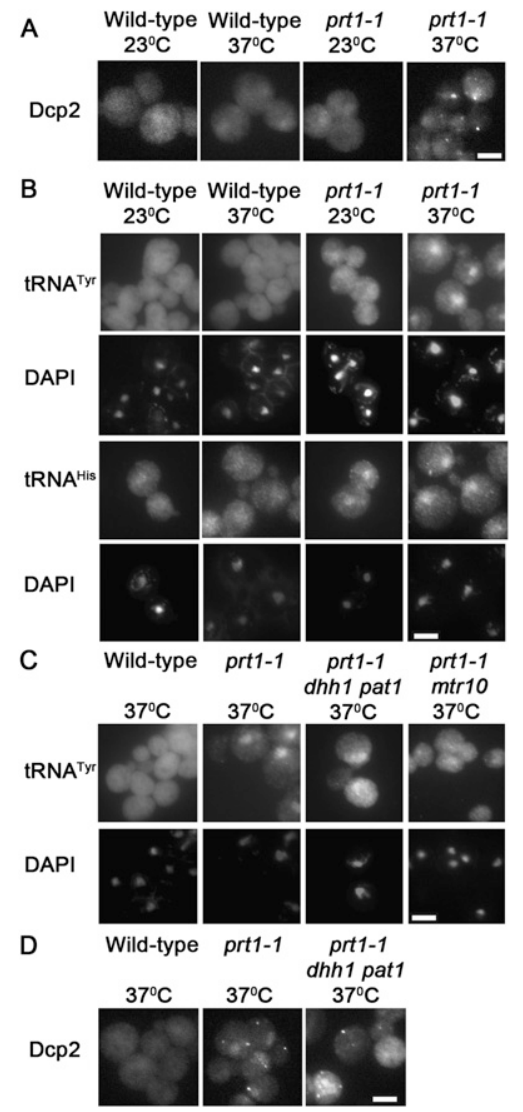

FIGURE 5. tRNA nuclear accumulation observed in prt1-1 cells is partially Dhh1/Pat1-dependent. (A) Distribution of Dcp2-RFP within wild-type and prt1-1 cells grown at $23^{\circ} \mathrm{C}$ or incubated at $37^{\circ} \mathrm{C}$ for $2 \mathrm{~h}$. (B) Wild-type and prt1-1 cells grown at $23^{\circ} \mathrm{C}$ or incubated at $37^{\circ} \mathrm{C}$ for $2 \mathrm{~h}$ were analyzed by FISH to determine the distribution of tRNA ${ }^{\text {Tyr }}$ (row 1), tRNA $^{\text {His }}$ (row 3), and DNA (DAPI; rows 2,4). These results were confirmed in the A364 background (data not shown). (C) Distribution of $\mathrm{tRNA}^{\mathrm{Tyr}}$ (row 1) and DNA (DAPI; row 2) within wild-type (BY4741), prt1-1 (B. prt1-1), prt1-1 mtr10, and prt1-1 dhh1 pat 1 cells incubated at $37^{\circ} \mathrm{C}$ for $2 \mathrm{~h}$ as determined by FISH. $(D)$ The distribution of plasmid-borne Dcp2-RFP within wild-type, prt1-1, prt1-1 dhh $1 \Delta$ pat $1 \Delta$ cells after $2 \mathrm{~h}$ incubation at $37^{\circ} \mathrm{C}$. Bars represent $5 \mu \mathrm{m}$.

was less than prt1-1 cells at nonpermissive temperature (Fig. 5C, column 2), but more than the nuclear levels of wild-type cells (Fig. 5C, column 1). We also observed that prt1-1 pat1s dhh1s cells formed Dcp2-RFP foci at nonpermissive temperature (Fig. $5 \mathrm{D}$, column 3 ). An interpretation of this result is that an additional pathway, that can bypass the need for Dhh1/Pat1, contributes to the tRNA nuclear accumulation induced by inhibition of translation initiation and P-body formation (Fig. 7A). In the case of the tRNA nuclear accumulation, this pathway is Mtr10dependent.

\section{DISCUSSION}

Cells initiate a variety of pathways in response to nutrient deprivation and cellular stresses. Coordinate regulation of these processes may allow cells to more effectively survive adverse conditions. This study documents that there is coordination between the early part of the P-body formation/mRNA decay/translation repression pathways and tRNA nuclear-cytoplasmic distribution (Fig. 7). Dhh1 and Pat1 function, in parallel, to promote starvation-induced tRNA nuclear accumulation as they also do for P-body formation and translation repression (Fig. 7B; Holmes et al. 2004; Coller and Parker 2005). Either Dhh1 or Pat1 is sufficient to mediate tRNA nuclear accumulation in response to amino acid or glucose withdrawal as cells lacking both Dhh1 and Pat1 fail to exhibit starvationinduced tRNA nuclear accumulation, whereas single deletions of either DHH1 or PAT1 are able to do so. Similarly, overexpression of Dhh1 or Pat1 causes increased P-body formation, translation repression (Coller and Parker 2005), and tRNA nuclear accumulation. The requirement for either Dhh1 or Pat1 to mediate amino acid starvationinduced tRNA nuclear accumulation highlights the largely understudied roles of Dhh1 and Pat1 in an amino acid starvation response. Previous studies demonstrated that

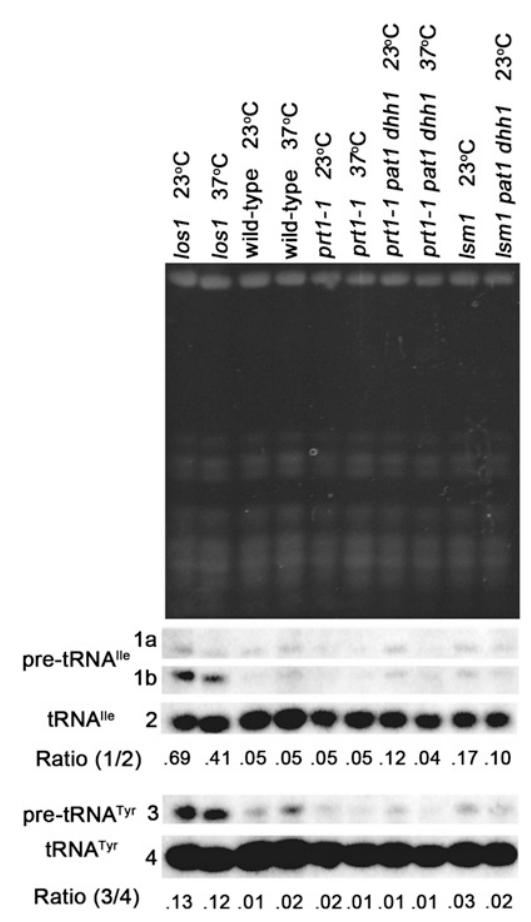

FIGURE 6. The tRNA nuclear accumulation observed in prt1-1 cells is due to nuclear import and/or defective re-export. Northern analysis of RNA extracted from wild-type, prt1-1, prt1-1 pat $1 \mathrm{dhh} 1, \mathrm{lsm} 1, \mathrm{lsm} 1$ pat $1 \mathrm{dhh} 1$, and los 1 cells grown at $23^{\circ} \mathrm{C}$ or incubated at $37^{\circ} \mathrm{C}$ for $2 \mathrm{~h}$ The los 1 extracts provide intron-containing pre-tRNA accumulation (positive) controls. Ethidium bromide staining of gel (top panel) prior to transfer reveals the RNAs present and serves to assess the amount of RNA loaded in each well. Blots were probed with ${ }^{32} \mathrm{P}$-labeled oligonucleotides that hybridized to tRNA ${ }^{\text {Ile }}$ (1a-initial transcript of pre-tRNA ${ }^{\text {Ile }} ; 1 b$-end-processed, intron-containing pre-tRNA ${ }^{\text {Ile }}$, 2-mature tRNA ${ }^{\text {Ile }}$ ) or tRNA ${ }^{\text {Tyr }}$ (3-precursor tRNA ${ }^{\text {Tyr }}$; 4-mature tRNA $\left.^{\text {Tyr }}\right)$. Ratios of pre-tRNA to mature tRNA are presented. 


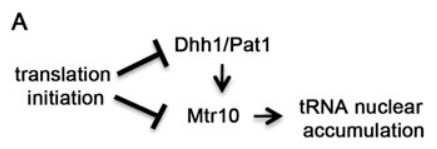

B

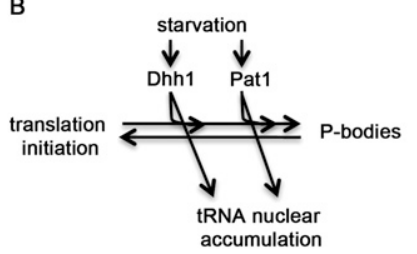

FIGURE 7. Model for the roles of Dhh1, Pat1, and translation initiation in the regulation of tRNA nucleus-cytosol distribution. (A) Translation initiation promotes normal distribution of tRNA. Loss of translation initiation via the prt1-1 mutation causes tRNA to accumulate within nuclei through Dhh1/Pat1-dependent and -independent pathways. (B) Cells experiencing acute starvation stimulate Dhh1 and Pat1 to promote tRNA nuclear accumulation. As Dhh1 and Pat1 are functioning in parallel, loss of either protein will not block starvation-induced tRNA nuclear accumulation, P-body formation, or translation repression.

amino acid starvation-induced translation repression is impaired in $d h h 1$ and pat 1 cells (Holmes et al. 2004), and is blocked in dhh1 pat1 cells (Coller and Parker 2005). This suggests that Dhh1 and Pat1 may have an intermediate level of function that allows them to repress translation and induce tRNA nuclear accumulation without P-body formation, or that there may be a separate pathway that prevents aggregation of P-body components when glucose is available. The data indicate that Dhh1 and Pat1 also have parallel roles in amino acid and glucose responsive tRNA subcellular distribution dynamics.

Additional P-body/mRNA decay components were evaluated to determine if they also affect tRNA distribution. The tRNA nuclear accumulation observed in fed $l s m 1 \Delta$ cells provides an additional connection between tRNA nuclear-cytoplasmic distribution and P-body formation. The mechanism by which this tRNA accumulation occurs may become clear as the mechanism by which $l s m 1 \Delta$ causes increased P-body formation is elucidated. Consistent with Dhh1 and Pat1 having roles in this process, the triple mutant, pat1s dhh1s $l s m 1 \Delta$ cells did not exhibit tRNA nuclear accumulation.

Coordination between tRNA distribution and P-body formation is limited to the early steps of these pathways as loss of the decapping activiation domain from Pat1 (pat1 254-422) or loss of mRNA decapping (dcp2-7) does not affect tRNA nuclear-cytoplasmic dynamics. Similarly, loss of $5^{\prime}$ to $3^{\prime}$ decay $(x r n 1 \Delta)$ does not cause nuclear accumulation of tRNA. Therefore, tRNA nuclear-cytoplasmic distribution is coordinated with early steps of P-body formation in a Dhh1/Pat1-dependent manner during starvation.

Investigation of tRNA nuclear-cytoplasmic dynamics of the temperature-sensitive, eIF3 subcomponent, prt1-1, provided data supporting a tRNA nuclear accumulation pathway that may be independent of Dhh1 or Pat1, but dependent on Mtr10, which is involved in tRNA import (Fig. 7A). This is in addition to the previously demonstrated increase in mRNA decay (Barnes et al. 1993), increase in P-body formation (Brengues et al. 2005), and disruption of translation initiation (Barnes et al. 1993) in prt1-1 cells. It is surprising that the tRNA nuclear accumulation observed in prt1-1 cells is only partially dependent on Dhh1 and Pat1 as tRNA nuclear accumulation is reduced, but not eliminated, in prt1-1 pat $1 \Delta$ dhh1 1 cells at the nonpermissive temperature. However, the prt1-1 mtr10s cells exhibit an even distribution of tRNA at the nonpermissive temperature indicating that the tRNA nuclear accumulation caused by defects in translation initiation requires tRNA nuclear import. This conclusion is supported by the Northern analysis of prt1-1 cells, which shows that the tRNA accumulating within the nuclei are spliced. The residual tRNA accumulation observed in prt1-1 pat1s dhh1s cells grown at nonpermissive temperature may be due to a Dhh1/Pat1-independent pathway(s) or secondary effect of inhibiting translation initiation.

Since there is detectable nuclear accumulation of tRNA within prt1-1 pat1s dhh1s cells at nonpermissive temperature, pat $1 \Delta$ dhh1s cells must not be completely blocked for tRNA nuclear import. The loss of starvation-induced tRNA nuclear accumulation in pat $1 \Delta d h h 1 \Delta$ cells may be due to an inability of the cells to retain tRNA within nuclei. This suggests that Pat1 and Dhh1 may participate in regulatory pathways that alter rates of tRNA re-export and/or import, resulting in starvation-induced tRNA nuclear accumulation.

The current model is that Dhh1 and Pat1 function in parallel to promote tRNA nuclear accumulation when deprived of nutrients (Fig. 7B). One possible mechanism of action is that Dhh1/Pat1 are bifunctional proteins that not only function in mRNA-related activities (translation/ P-body formation/stress granules), but are also part of a signaling cascade that results in starvation-induced tRNA nuclear accumulation. A second possible mechanism is that cells detect early steps of the Dhh1/Pat1 mediated responses to nutrient loss, and signal for tRNA nuclear accumulation. This model predicts that loss of Dhh1 and Pat1 (pat1s $d h h 1 \Delta$ ) would block the signaling for starvation-induced tRNA nuclear accumulation without disrupting the basal level of retrograde import and/or re-export of tRNA. These and other implications of this model will be addressed in future studies.

While this work has demonstrated that there is coordination of P-body formation, translation initiation, and tRNA nuclear-cytoplasmic dynamics in response to nutrient availability, many questions remain to be addressed. The mechanism(s) by which cells sense the nutrient loss and then alter Pat 1 and Dhh1 activity has yet to be elucidated. The rapid response time suggests post-translational modifications. There may be different modifications resulting 
from loss of each nutrient. The mechanism(s) utilized by Pat1 and Dhh1 to influence tRNA nuclear-cytoplasmic distribution has yet to be uncovered. As Dhh1 and Pat1 have RNA binding domains and are not excluded from the nucleus (Teixeira and Parker 2007), direct transport of tRNA from the cytoplasm to the nucleus by Dhh1 and Pat1 is possible. However, this direct mode seems unlikely because Pat1 and Dhh1 aggregate in the cytoplasm when cells are starved, in contrast to the nuclear accumulation of the tRNA. An alternative possibility is that Dhh1 and Pat1 participate in a signaling pathway thereby indirectly regulating tRNA nuclear-cytoplasmic distribution. Addressing these possibilities may provide insight into what advantage or benefit does relocating tRNA to the nucleus during starvation provide for the cell.

\section{MATERIALS AND METHODS}

\section{Plasmids and strains}

The strains used in this study are listed in Supplemental Table 2. All yeast transformations were done by standard lithium acetate procedures. Plasmids used in this study are listed in Supplemental Table 3. The PAT1/DHH1 containing plasmid, pRPDhh1-16, was made by ligating the Dhh1-GFP containing fragment from BamHI-digested pDHH1018 to BamHI-digested pRP1478.

Yeast deletions were accomplished by transformation of a parent strain with a knockout cassette designed to replace the endogenous gene with a selection marker (Goldstein and McCusker 1999; Gueldener et al. 2002). Sequences of the oligonucleotides used for this study are in Supplemental Table 4. The knockout cassettes were generated by PCR amplification using oligonucleotides where the first ( $5^{\prime}$ to $3^{\prime}$ ) 50-70 bases targeted the product to the upstream of or downstream from the genomic region encoding the gene to be removed by homologous recombination, and the last 18-22 bases allowed base pairing of the oligonucleotides to the template containing the selection marker. The template and oligonucleotide combinations used are described in Supplemental Table 5.

The pat1ko8, dhh1ko1, xrn1ko8, and lsm1ko6 strains were generated by transformation of BY4741 with pat1::natMX4, dhh1::hphMX4, kem1::hphMX4, and lsm $1:: K l$. leu2, respectively. Transformation of pat 1 ko 8 with $d h h 1:: h p h M X 4$ resulted in pat1dhh1-11. Subsequent transformations of pat1dhh1-11 with lsm $1:: K l$. leu 2 and $\operatorname{trp} 1:: K l$. leu2 produced pat1 dhh1 lsm1 (pdl-B; Supplemental Table 2) and pat1 dhh1 trp1 (pdt-1; Supplemental Table 2) strains, respectively. The prt1-1 mtr10 (B. prt1-1 mtr10-8; Supplemental Table 2) strain was generated by transformation of prt1-1 (B. prt1-1 in Supplemental Table 2) with mtr10::natMX4. The prt1-1 dhh1 pat1 (pdHEpLB; Supplemental Table 2) strain was made by transformation of B. prt1-1 with pRPDhh1-16, followed by dhh1::hphMX4, then pat $1:: K l$. leu2, and finally the pRPDhh1-16 was lost using a 5FOA counter-selection against the URA3 encoded on the plasmid.

To select for natMX4 and/or hphMX4 cassette integration in nutrient-rich conditions, the strains were grown on YEPD (yeast extract, trypticase peptone, dextrose, supplemented with adenine $[0.04 \mathrm{~g} / \mathrm{L}]$, uracil $[0.04 \mathrm{~g} / \mathrm{L}])$, agar $[20 \mathrm{~g} / \mathrm{L}]$ with clonNAT $(100$ $\mathrm{mg} / \mathrm{L}$; Werner BioAgents, Jena, Germany) and/or hygromycin B
(300 mg/L; Calbiochem, La Jolla, CA). To select for natMX4 or $h p h M X 4$ cassette integration in the absence of uracil, the strains were grown on SCB -ura (Synthetic Complete Boone defined medium; Difco yeast nitrogen base without amino acids and ammonium sulfate [1.7 g/L; Becton, Dickinson, and Company, Sparks, MD] supplemented with amino acids, dextrose [2\%], adenine $[0.04 \mathrm{~g} / \mathrm{L}]$, monosodium glutamate $[1.0 \mathrm{~g} / \mathrm{L}])$ with agar $(20 \mathrm{~g} / \mathrm{L})$ and either clonNAT $(100 \mathrm{mg} / \mathrm{L})$ or hygromycin B $(300$ $\mathrm{mg} / \mathrm{L}$ ). To select for $K$. lactis LEU2 cassette integration, the strains

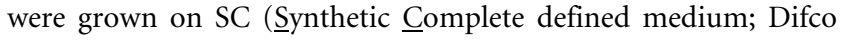
yeast nitrogen base without amino acids $[6.7 \mathrm{~g} / \mathrm{L}$; Becton, Dickinson, and Company, Sparks, MD] supplemented with amino acids, dextrose $[2 \%]$, adenine $[0.04 \mathrm{~g} / \mathrm{L}]$, and uracil $[0.04 \mathrm{~g} / \mathrm{L}]$ ) lacking leucine and with agar $(20 \mathrm{~g} / \mathrm{L})$ added.

Correct insertions of knockout cassettes were verified by PCR amplification of genomic DNA using combinations of oligonucleotides complementary to regions upstream of, downstream from, and/or midstream to the targeted gene and/or oligonucleotides complementary to the midstream of the selection marker (Supplemental Table 5).

\section{Fluorescence in situ hybridization and Northern analysis}

Cells were grown in liquid SC or SC variations lacking bases (e.g., SC-ura), amino acids (e.g., SC-aa), or dextrose (SC-glu). SCura+raff liquid medium is SC lacking dextrose and uracil with addition of $2 \%$ raffinose. SC-aa+gal liquid medium is SC lacking all amino acids and dextrose with addition of $2 \%$ galactose. Cells were grown in $15 \mathrm{~mL}$ of SC media for $10-14 \mathrm{~h}$ at $23^{\circ} \mathrm{C}$ to $A_{600}$ between 0.15 and 0.30 . For starvation experiments, cells from 15 $\mathrm{mL}$ cultures $\left(A_{600}\right.$ between 0.15 and 0.30 , use unless otherwise noted) were pelleted, the media was removed, the cells were resuspended in $5 \mathrm{~mL}$ of starvation media (SC-aa, or SC-glu), cells were re-pelleted, the media was removed, the cells were resuspended in $15 \mathrm{~mL}$ of starvation media (SC-aa, or SC-glu), and the suspension returned to the $23^{\circ} \mathrm{C}$ shaking water bath for $2 \mathrm{~h}$ prior to use. Galactose induction of cultures was accomplished by the addition of $1 / 10$ volume of $20 \%$ galactose to cells $\left(A_{600}\right.$ between 0.12 and 0.15 ) growing in media containing raffinose as the carbon source $3 \mathrm{~h}$ prior to use.

RNA extraction from low-density cultures $\left(A_{600} 0.15-0.3\right)$ and subsequent Northern analysis was performed as previously described (Hopper et al. 1980) with the previously described modifications (Hurto et al. 2007). Fluorescence in situ hybridization was performed as previously described (Sarkar and Hopper 1998) with the previously described modifications (Stanford et al. 2004). Each slide contained positive and negative controls for tRNA nuclear accumulation. When adjectives describing the relative amount of tRNA nuclear accumulation are utilized, the probes were hybridized under the same conditions in different wells on the same slide. All critical experiments were independently viewed and scored by at least two people, one of whom was unaware of the experimental details.

\section{Visualization of P-body markers}

To capture the low-density phenotype and avoid oxidative stress, the cells were fixed in the same manner as used for FISH. Cells were cultured ( $A_{600}$ between 0.2 and 0.4 ) and fixed as described 
earlier. After treatment with zymolyase $(\sim 20 \mathrm{~min})$ in buffer $(1.2$ M sorbitol, $0.1 \mathrm{M} \mathrm{KHPO} 4 \mathrm{pH} 6.5$ ), the cells were collected by centrifugation. Cells were resuspended in fresh buffer and then collected by centrifugation. The cells were resuspended in the residual buffer $(\sim 250 \mu \mathrm{L})$ and placed on poly-L-lysine coated slides. Cells were dehydrated with $70 \%, 90 \%$, and $100 \%$ ethanol washes. Mounting media was applied and slides were sealed. This procedure worked for the visualization of Edc3-mCherry and Dcp2RFP. However, Lsm1-RFP containing aggregates were undetectable using this procedure.

The small size of the Dcp2 and Edc3 foci is not unexpected for several reasons. First, cells from lower density cultures have been previously shown to have smaller foci (Teixeira et al. 2005). Second, the starvation was a transition from synthetic media to synthetic media lacking glucose. This is in contrast to the frequently used transition from YEPD to synthetic media lacking glucose or water which is known to produce large sized P-bodies (Nissan and Parker 2008). Additionally, the fixation process or protracted length of deprivation ( $2 \mathrm{~h}$ vs. $10 \mathrm{~min}$ ) may have reduced the intensity of fluorescence.

Microscopy and image capture was accomplished using 60X and 100X objectives with either a Nikon Microphot-FX microscope with a Sensys charge-coupled device camera (Photometrics, Tucson, AZ) using QED software (QED Imaging, Pittsburgh, PA) or a Nikon 90i equipped with a CoolSNAP HQ2 digital camera and MetaMorph software (Molecular Devices, Sunnyvale, CA) or NIS-Elements (Nikon, Melville, NY). Images were assembled using Adobe Photoshop (Adobe Systems, San Jose, CA).

\section{SUPPLEMENTAL MATERIAL}

Supplemental material is available for this article.

\section{ACKNOWLEDGMENTS}

We are very grateful for the many plasmids and strains provided by R. Parker (University of Arizona). We thank C. Boone (University of Toronto, Canada) for the B. prt1-1 strain (in Supplemental Table 2). We also thank the members of the A. K. Hopper Lab, J. Larson, and V. Ramachandran for comments on the manuscript. This work was supported by The National Institutes of Health grant no. GM-27930 (to A.K.H.).

Received November 21, 2010; accepted February 6, 2011.

\section{REFERENCES}

Arts GJ, Fornerod M, Mattaj IW. 1998a. Identification of a nuclear export receptor for tRNA. Curr Biol 8: 305-314.

Arts GJ, Kuersten S, Romby P, Ehresmann B, Mattaj IW. 1998b. The role of exportin-t in selective nuclear export of mature tRNAs. EMBO J 17: 7430-7441.

Barbet NC, Schneider U, Helliwell SB, Stansfield I, Tuite MF, Hall MN. 1996. TOR controls translation initiation and early G1 progression in yeast. Mol Biol Cell 7: 25-42.

Barnes CA, Singer RA, Johnston GC. 1993. Yeast prt1 mutations alter heat-shock gene expression through transcript fragmentation. EMBO J 12: 3323-3332.

Bohnsack MT, Regener K, Schwappach B, Saffrich R, Paraskeva E, Hartmann E, Gorlich D. 2002. Exp5 exports eEF1A via tRNA from nuclei and synergizes with other transport pathways to confine translation to the cytoplasm. EMBO J 21: 6205-6215.

Brengues M, Teixeira D, Parker R. 2005. Movement of eukaryotic mRNAs between polysomes and cytoplasmic processing bodies. Science 310: 486-489.

Buchan JR, Parker R. 2009. Eukaryotic stress granules: the ins and outs of translation. Mol Cell 36: 932-941.

Buchan JR, Nissan T, Parker R. 2010. Analyzing P-bodies and stress granules in Saccharomyces cerevisiae. Methods Enzymol 470: 619-640.

Calado A, Treichel N, Muller EC, Otto A, Kutay U. 2002. Exportin5-mediated nuclear export of eukaryotic elongation factor $1 \mathrm{~A}$ and tRNA. EMBO J 21: 6216-6224.

Cheng Z, Coller J, Parker R, Song H. 2005. Crystal structure and functional analysis of DEAD-box protein Dhhlp. RNA 11: 12581270.

Coller J, Parker R. 2005. General translational repression by activators of mRNA decapping. Cell 122: 875-886.

Cook AG, Fukuhara N, Jinek M, Conti E. 2009. Structures of the tRNA export factor in the nuclear and cytosolic states. Nature 461: 60-65.

Eswara MB, McGuire AT, Pierce JB, Mangroo D. 2009. Utp9p facilitates Msn5p-mediated nuclear reexport of retrograded tRNAs in Saccharomyces cerevisiae. Mol Biol Cell 20: 5007-5025.

Goldstein AL, McCusker JH. 1999. Three new dominant drug resistance cassettes for gene disruption in Saccharomyces cerevisiae. Yeast 15: 1541-1553.

Grosshans H, Hurt E, Simos G. 2000. An aminoacylation-dependent nuclear tRNA export pathway in yeast. Genes Dev 14: 830-840.

Gueldener U, Heinisch J, Koehler GJ, Voss D, Hegemann JH. 2002. A second set of loxP marker cassettes for Cre-mediated multiple gene knockouts in budding yeast. Nucleic Acids Res 30: e23. doi: 10.1093/nar/30.6.e23.

Hellmuth K, Lau DM, Bischoff FR, Kunzler M, Hurt E, Simos G. 1998. Yeast Los1p has properties of an exportin-like nucleocytoplasmic transport factor for tRNA. Mol Cell Biol 18: 63746386.

Holmes LE, Campbell SG, De Long SK, Sachs AB, Ashe MP. 2004. Loss of translational control in yeast compromised for the major mRNA decay pathway. Mol Cell Biol 24: 2998-3010.

Hopper AK, Schultz LD, Shapiro RA. 1980. Processing of intervening sequences: a new yeast mutant which fails to excise intervening sequences from precursor tRNAs. Cell 19: 741-751.

Hunter CA, Aukerman MJ, Sun H, Fokina M, Poethig RS. 2003. PAUSED encodes the Arabidopsis exportin-t ortholog. Plant Physiol 132: 2135-2143.

Hurto RL, Tong AH, Boone C, Hopper AK. 2007. Inorganic phosphate deprivation causes tRNA nuclear accumulation via retrograde transport in Saccharomyces cerevisiae. Genetics 176: 841-852.

Kutay U, Lipowsky G, Izaurralde E, Bischoff FR, Schwarzmaier P, Hartmann E, Gorlich D. 1998. Identification of a tRNA-specific nuclear export receptor. Mol Cell 1: 359-369.

Lipowsky G, Bischoff FR, Izaurralde E, Kutay U, Schafer S, Gross HJ, Beier H, Gorlich D. 1999. Coordination of tRNA nuclear export with processing of tRNA. RNA 5: 539-549.

Lund E, Dahlberg JE. 1998. Proofreading and aminoacylation of tRNAs before export from the nucleus. Science 282: 2082-2085.

Murthi A, Shaheen HH, Huang HY, Preston MA, Lai TP, Phizicky EM, Hopper AK. 2010. Regulation of tRNA bidirectional nuclearcytoplasmic trafficking in Saccharomyces cerevisiae. Mol Biol Cell 21: 639-649.

Nielsen KH, Szamecz B, Valasek L, Jivotovskaya A, Shin BS, Hinnebusch AG. 2004. Functions of eIF3 downstream of $48 \mathrm{~S}$ assembly impact AUG recognition and GCN4 translational control. EMBO J 23: 1166-1177.

Nissan T, Parker R. 2008. Analyzing P-bodies in Saccharomyces cerevisiae. Methods Enzymol 448: 507-520.

Nissan T, Rajyaguru P, She M, Song H, Parker R. 2010. Decapping activators in Saccharomyces cerevisiae act by multiple mechanisms. Mol Cell 39: 773-783. 
Pemberton LF, Rosenblum JS, Blobel G. 1997. A distinct and parallel pathway for the nuclear import of an mRNA-binding protein. J Cell Biol 139: 1645-1653.

Phizicky EM, Hopper AK. 2010. tRNA biology charges to the front. Genes Dev 24: 1832-1860.

Pilkington GR, Parker R. 2008. Patl contains distinct functional domains that promote P-body assembly and activation of decapping. Mol Cell Biol 28: 1298-1312.

Sarkar S, Hopper AK. 1998. tRNA nuclear export in Saccharomyces cerevisiae: in situ hybridization analysis. Mol Biol Cell 9: 3041-3055.

Sarkar S, Azad AK, Hopper AK. 1999. Nuclear tRNA aminoacylation and its role in nuclear export of endogenous tRNAs in Saccharomyces cerevisiae. Proc Natl Acad Sci 96: 14366-14371.

Shaheen HH, Hopper AK. 2005. Retrograde movement of tRNAs from the cytoplasm to the nucleus in Saccharomyces cerevisiae. Proc Natl Acad Sci 102: 11290-11295.

Shaheen HH, Horetsky RL, Kimball SR, Murthi A, Jefferson LS, Hopper AK. 2007. Retrograde nuclear accumulation of cytoplasmic tRNA in rat hepatoma cells in response to amino acid deprivation. Proc Natl Acad Sci 104: 8845-8850.

Sheth U, Parker R. 2003. Decapping and decay of messenger RNA occur in cytoplasmic processing bodies. Science 300: 805-808.

Shibata S, Sasaki M, Miki T, Shimamoto A, Furuichi Y, Katahira J, Yoneda Y. 2006. Exportin-5 orthologues are functionally divergent among species. Nucleic Acids Res 34: 4711-4721.

Spriggs KA, Bushell M, Willis AE. 2010. Translational regulation of gene expression during conditions of cell stress. Mol Cell 40: 228-237.
Stanford DR, Whitney ML, Hurto RL, Eisaman DM, Shen W-C, Hopper AK. 2004. Division of labor among the yeast Sol proteins implicated in tRNA nuclear export and carbohydrate metabolism. Genetics 168: 117-127.

Takano A, Endo T, Yoshihisa T. 2005. tRNA actively shuttles between the nucleus and cytosol in yeast. Science 309: 140-142.

Teixeira D, Parker R. 2007. Analysis of P-body assembly in Saccharomyces cerevisiae. Mol Biol Cell 18: 2274-2287.

Teixeira D, Sheth U, Valencia-Sanchez MA, Brengues M, Parker R. 2005. Processing bodies require RNA for assembly and contain nontranslating mRNAs. RNA 11: 371-382.

Tharun S, Parker R. 2001. Targeting an mRNA for decapping: displacement of translation factors and association of the Lsmlp7p complex on deadenylated yeast mRNAs. Mol Cell 8: 1075-1083.

Whitney ML, Hurto RL, Shaheen HH, Hopper AK. 2007. Rapid and reversible nuclear accumulation of cytoplasmic tRNA in response to nutrient availability. Mol Biol Cell 18: 2678-2686.

Yoshihisa T, Yunoki-Esaki K, Ohshima C, Tanaka N, Endo T. 2003. Possibility of cytoplasmic pre-tRNA splicing: the yeast tRNA splicing endonuclease mainly localizes on the mitochondria. Mol Biol Cell 14: 3266-3279.

Yoshihisa T, Ohshima C, Yunoki-Esaki K, Endo T. 2007. Cytoplasmic splicing of tRNA in Saccharomyces cerevisiae. Genes Cells 12: 285297.

Zaitseva L, Myers R, Fassati A. 2006. tRNAs promote nuclear import of HIV-1 intracellular reverse transcription complexes. PLoS Biol 4: e332. doi: 10.1371/journal.pbio.0040332. 

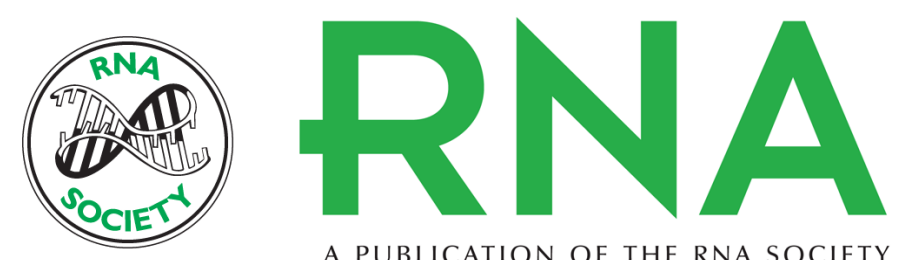

A PUBLICATION OF THE RNA SOCIETY

\section{P-body components, Dhh1 and Pat1, are involved in tRNA nuclear-cytoplasmic dynamics}

Rebecca L. Hurto and Anita K. Hopper

RNA 2011 17: 912-924 originally published online March 11, 2011

Access the most recent version at doi:10.1261/rna.2558511

\section{Supplemental http://rnajournal.cshlp.org/content/suppl/2011/03/01/rna.2558511.DC1 \\ Material}

References This article cites 47 articles, 28 of which can be accessed free at: http://rnajournal.cshlp.org/content/17/5/912.full.html\#ref-list-1

\section{License}

Email Alerting Receive free email alerts when new articles cite this article - sign up in the box at the Service top right corner of the article or click here.

To subscribe to $R N A$ go to:

http://rnajournal.cshlp.org/subscriptions 